\title{
Escala multidimensional de satisfação de vida para crianças: estudos de construção e validação
}

\section{Multidimensional life satisfaction scale for children: development and validation studies}

\author{
Claudia Hofheinz GIACOMONI ${ }^{1}$ \\ Cláudio Simon HUTZZ
}

\begin{abstract}
Resumo
Os objetivos deste trabalho foram desenvolver uma escala, que foi intitulada de Escala Multidimensional de Satisfação de Vida para Crianças, assim como apresentar informações substanciais sobre as propriedades psicométricas do instrumento. Paralelamente, viabilizou-se a testagem do Modelo Multidimensional de Satisfação de Vida Infantil de Huebner. Foram realizados estudos de construção e de validação da escala. A versão final da Escala Multidimensional de Satisfação de Vida para Crianças contém 50 itens, distribuídos em seis fatores: self, self Comparado, Não-Violência, Família, Amizade e Escola. Foram encontradas consistências internas adequadas para cada uma das sub-escalas, assim como para a escala total. A validade concorrente das sub-escalas da escala multidimensional de satisfação de vida para crianças foi confirmada por correlações médias com medidas critério.
\end{abstract}

Unitermos: Bem-estar subjetivo. Escala de satisfação de vida. Validade.

\begin{abstract}
The main purpose of this study was to develop a Multidimensional Life Satisfaction Scale for Children, as well as to present significant psychometric information. In parallel, wetested Huebner's Children's Life Satisfaction Multidimensional Model. Construction and validation studieswerecarried out. Thefinal version of the Multidimensional LifeSatisfaction ScaleforChildren includes50items, divided into 6 groupings: self,Compared self, Non-violence, Family, Friendship and School. Wefound adequateinternal consistenciesforeach subscaleaswell asforthe scale as a whole. The concurrent validity of the Children's Life Satisfaction Multidimensional sub-scales was confirmed through average correlation coefficients using criterion measurement.
\end{abstract}

Uniterms: Subjective well-being. Life satisfaction scale. Validity.

\footnotetext{
${ }^{1}$ UniversidadeFederal deSanta Maria,Curso de Psicologia.Av. Roraima, 1000, Cidade Universitária, Camobi,97105-900,Santa Maria, RS,Brasil.Correspondência para/Correspondence to: C.H. GIACOMONI.E-mail: <giacomon@uol.com.br>.

2 Universidade Federal do Rio Grande do Sul, Instituto de Psicologia, Departamento de Psicologia do Desenvolvimento e da Personalidade. Porto Alegre, RS, Brasil.

Apoio: Coordenação de Aperfeiçoamento de Pessoal de Nível Superior.

Agradecimento aosalunos do Curso de Psicologia da Universidade Federal do Rio Grande do Sul:Ana Paula Tibulo, Gabriel da Silva Mazzini e J oceline Fátima Zanchetin, pela participação na coleta, digitação e análise dos dados.
} 
O bem-estar subjetivo é composto por trêsfatores, que se inter-relacionam: 0 afeto positivo, 0 afeto negativo e a satisfação de vida (Andrews \& Withey, 1976; Campbell, Converse \& Rodgers, 1976; Diener 1984, 1994). Os dois primeiros são definidos pelas respostas afetivas, enquanto a satisfação de vida é definida como uma resposta avaliativa cognitiva. Mais especificamente, a avaliação da satisfação de vida pode ser feita globalmente, ou seja, da vida como um todo, ou sobre domínios específicos, como, por exemplo, a família, o trabalho, a vida escolar.

Além das medidas sobre satisfação de vida global (Diener, Emmons, Larsen \&Griffin, 1985), algumas medidas multidimensionais têm sido desenvolvidas para o uso em pesquisas com adultos (Evans, Burns, Robinson \& Garret, 1985; Frisch, Cornell, Villanueva \& Retzlaff, 1992). Medidas especialmente desenvolvidas para crianças são mais raras do que para adultos.

Encontrou-se, na literatura, duas escalas que avaliam satisfação de vida global infantil: a Escala de Satisfação de Vida Percebida, de Adelman, Taylore Nelson (1989), e a Escala de Satisfação de Vida de Estudantes, de Huebner (1991a, 1994b, 1995), além de escalas para avaliar qualidade de vida infantil (Assumpção, Kuczynski, Sprovieri \& Aranha, 2000; Harding, 2001).

Alguns anos após a construção das primeiras escalas unidimensionais de satisfação de vida, foi desenvolvida a Escala de Satisfação de Vida Multidimensional, por Huebner (Huebner,1994a, 1998a, 1998b; Huebner, Laughlin, Ash \&Gilman,1998; Gilman, Huebner $\&$ Laughlin, 2000), baseada nos modelos multidimensionais explicativos de bem-estar subjetivo de adultos (Headey, Holmstrom \& Wearing, 1984) e nos resultados dos estudos correlatos entre satisfação de vida infantile outras variáveis. Huebner também propôs o Modelo Multidimensional de Satisfação de Vida Infantil (Huebner, 1994a), no qual constavam os seguintes domínios: família, escola, self e amizade. 0 que levou Huebner a criar este modelo foi o fato de os estudos já realizados (Huebner, 1998a, 1998b; Huebner et al., 1998; Huebner \& Dew, 1993; Gilman et al., 2000), em que se utilizou a Escala de Satisfação de Vida Multidimensional, sugerirem que crianças e adolescentes diferenciam, assim como os adultos, os diferentes domínios de satisfação de vida.
No Brasil, encontra-se a adaptação da Escala de Satisfação de Vida de Estudantes, por Giacomoni (1998), com resultadosnão satisfatórios quanto à confiabilidade (Alpha de Cronbach $=0,50$ ). Entre as hipóteses analisadas por este autor como causa da baixa fidedignidade encontrada para esta escala de Huebner, é apontada a influência dos aspectos culturais, que determinam a constituição das crenças, valores e metas individuais que, por sua vez, também compõem o processo de avaliação da vida.

A partir dos resultados de outro estudo de Giacomoni (2002) sobre o conceito de felicidade, ampliou-se o Modelo Multidimensional de Satisfação de Vida Infantil de Huebner, adicionando-se, aos quatro domínios já existentes, outros quatro novos domínios: satisfação de necessidades básicas materiais, lazer, não-violência e satisfação de desejos.

Observando a falta de instrumentos adequadamente elaborados para crianças e desenvolvidos a partir da cultura brasileira, e buscando testar o Modelo Multidimensional de Satisfação de Vida Infantil, definiu-se como objetivo geral deste estudo desenvolver uma Escala Multidimensional de Satisfação de Vida para Crianças (EMSVC). Além disso, procurou-se: a) identificar um perfil da satisfação das crianças com relação aos domínios específicos de suas vidas; b) verificar as características psicométricas do instrumento construído; c) verificar se a estrutura fatorial encontrada na escala multidimensional envolve dimensões psicologicamente significativas; d) comprovar a validade concorrente do instrumento, pela comparação com instrumentos que medem construtos psicológicos correlatos; e) verificar, quanto aos domínios de satisfação de vida infantil, possíveis diferenças entre sexos, faixa etária e tipo de escola. Para tanto, foram realizados dois estudos, apresentados a seguir: um de construção da escala e outro com intuito de validá-la.

\section{Método}

Tanto na construção da escala quanto em sua validação, houve a participação de crianças, pois 0 instrumento foi elaborado em função dos resultados parciais obtid os. Primeiramente, elaborou-se uma versão preliminar, com base em diversas escalas já existentes, que foi mostrada para um pequeno grupo de crianças 
( $n=10) ;$ e, considerando-se a dificuldade das crianças frente a alguns itens, os mesmos foram reelaborados ou excluídos, chegando-se a uma segunda versão, com 60 itens. Então, selecionou-se as escolas para a realização da pesquisa, e aplicou-se a escala à amostra completa de crianças do Estudo 1 ( $n=661$ ). Apenas na etapa de análise de resultados, o número de itens foi reduzido a 50. Então, teve início o segundo estudo, com o objetivo de validação do instrumento, com outra amostra de crianças $(n=230)$, utilizando-se instrumentos que avaliassem construtos correlatos ao bem-estar subjetivo e comparando-0s, posteriormente, aos resultados obtidos com a EMSVC. Os instrumentos utilizados, tanto aqueles em que se baseou a construção da EMSVC, quanto os que foram aplicados no segundo estudo para fins de comparação, estão descritos em Procedimentos dos Estudos 1 e 2, respectivamente.

Estudo 1: construção da escala multidimensional de satisfação de vida para crianças (EMSVC)

\section{Procedimentos}

A elaboração de itens e construção da versão preliminar da escala é apontada como a expressão da representação comportamental do construto que se pretende avaliar. A teoria sobre construção de instrumentos assinala como possíveis fontes de itens a entrevista, outros testes que medem o mesmo construto, e/ ou categorias comportamentais que expressem 0 construto de interesse. A entrevista consiste em investigar o tema junto aos sujeitos, representantes da população para a qual se deseja construir o instrumento (Pasquali, 1997, 1999).

Para a elaboração da EMSVC, os itens foram gerados a partir das entrevistas conduzidas no estudo de Giacomoni (2002), que apresenta o Modelo Multidimensional de Satisfação de Vida Infantil de Huebner, já com o total de oito domínios, sendo que tais entrevistas traziam perguntas sobre o conceito de felicidade e suas características, em crianças de cinco a doze anos. Além disso, também foram utilizados itens de outros instrumentos já validados por autores diversos.

Foram consultados instrumentos sobre bem-estar subjetivo infantil, e utilizou-se como possível fonte de modelos de itens a Escala de Satisfação de Vida de
Estudantes, também de Huebner (1991a), já adaptada para o português por Giacomoni (1998). Esta, inspirada no trabalho de Diener e colegas (Diener et al., 1985; Pavot, Diener, Colvin \& Sandvik, 1991), avalia a satisfação de vida global de crianças, e está baseada na hipótese de que a satisfação de vida global dessas é melhor avaliada por meio de itens que requeiram que elas avaliem as suas vidas como um todo, sem referências a domínios específicos. 0 estudante, para responder aos sete itens que compõem a escala, seleciona uma das quatro opções de freqüência: nunca (1), às vezes (2), geralmente (3) e quase sempre (4). As características psicométricas relatadas nos estudos anteriores demonstram que a escala é apropriada para os objetivos da pesquisa. 0 coeficiente Alpha encontrado no estudo original de Huebner (1991a) foi de 0,82.

Além da Escala de Satisfação de Vida de Estudantes, utilizou-se a Escala de Satisfação de Vida Multidimensional de Crianças (Huebner, 1994a, 1998a, 1998b; Hubner et al., 1998), que avalia as percepções subjetivas de satisfação de vida a partir de cinco domínios relevantes: família, amigos, escola, selfe ambiente onde vive (cinco sub-escalas compondo 40 itens no total). Estudos já desenvolvidos (Greenspoon \& Saklofske, 1998; Huebner, 1994a, 1998a, 1998b; Huebner et al., 1998) comprovam a boa consistência interna (Alpha de 0,92 ) e a estabilidade da estrutura fatorial.

Huebner (1991a, 1994a) identificou, entre os domínios de bem-estar subjetivo infantil: a família, a escola, a amizade, o self, as oportunidades de lazer e 0 ambiente onde vive. 0 estudo de Giacomoni (2002), que investigou o conceito de felicidade, suas características, os indicadores de qualidade de vida e os principais eventos positivos e negativos da vida infantil, propõe um novo modelo de satisfação de vida infantil, construído a partir da realidade do brasileiro. Assim, foram adicionados ao Modelo Multidimensional de Satisfação de Vida Infantil novos domínios de satisfação, finalizando-se com a seguinte estrutura: família, amizade, self, lazer, escola, não-violência, satisfação de necessidades básicas e de desejos. A partir desse modelo, discutido anteriormente,foram geradas sentenças (itens) para cada um dos domínios, assim como sentenças específicas sobre a satisfação total de vida infantil. Inicialmente, foram elaborados, aproximadamente, de 15 a 25 itens para cada domínio de satisfação de vida. 
Todo o processo de elaboração dos itens foi realizado por um grupo de pesquisa formado por pesquisadores em Psicologia do Desenvolvimento e por alunos de graduação em Psicologia. Após esta etapa, os itens foram analisados individualmente por cada membro da equipe de pesquisa, que elaborou um ranking dos itens considerados mais adequados e consistentes para o domínio específico, adotando-se critérios semânticose de ordem de preferência. Com a comparação dos rankings obtidos, foram selecionados os itens que obtiveram maior predileção. Após tal seleção, os itens finais foram analisados por um juiz que possui conhecimento na área.

Por sua vez, esses itens da primeira versão final foram mostrados para 10 crianças entre sete e doze anos de idade. As respostas das crianças indicavam o grau de concordância com o item, em uma escala de respostas do tipo Likert de cinco pontos, conforme 0 exemplo: "eu me acho uma pessoa bonita", e as possibilidades de resposta: (1) nem um pouco; (2) um pouco; (3) mais ou menos; (4) bastante e (5) muitíssimo. Com esse procedimento, foram verificados quais itens eram confusos, incompreensíveis, ambíguos e apresentavam problemas na sua construção. Também foi verificado o nível de compreensão da escala de resposta de cinco pontos.

Alguns itens foram reelaborados, principalmente aqueles que possuíam o sentido de resposta invertido, os chamados itens negativos. Foi verificado que as crianças não conseguiram responder de forma apropriada a esses itens. Este achado corrobora os de Marsh (1986), que verificou que os pré-adolescentes possuem inabilidade para responder de forma adequada a itens negativos, produzindo vieses de interpretação, devido a um fenômeno desenvolvimental-cognitivo. Huebner (1994a), em seus estudos de construção da sua Escala Multidimensional, encontrou a mesma dificuldade quanto ao uso de itens negativos com pré-adolescentes.

Diante da dificuldade das crianças frente aos itens invertidos na escala, optou-se pela eliminação de alguns desses do instrumento e pela inversão de sentido teórico de outros, apesar da indicação, pela teoria de construção de testes, da importância dositensnegativos. Os itens que possuíam semântica negativa e que foram invertidos, por sua vez, precisaram ter seus valores invertidos, posteriormente, na avaliação da escala, para que pudessem satisfazer ao sentido teórico da mesma.
Participantes e seleção das escolas

Após a construção da versão preliminar da escala, procedeu-se ao cálculo do número de participantes que deveria fazer parte da amostra. 0 número de participantes deste estudo foi calculado procurando atender ao critério da "razão itens/sujeito", geralmente utilizado para o cálculo amostral quando são necessárias análises fatoriais. Conforme tal critério, para que se possa realizar uma análise fatorial confiável, é importante que a amostra contenha pelo menoscinco vezes o número de itens do instrumento a ser avaliado. Pasquali (1999) recomenda que, quando não se tem certeza do número de dimensões ou fatores que o instrumento mede, deve-se trabalhar com uma amostra composta por 10 sujeitos por item. Sendo assim, como a escala relativa aos domínios possuía 60 itens, o número mínimo de participantes deveria ser de 600 crianças.

Finalmente, foram selecionadas as escolas onde se aplicaria a versão preliminardo instrumento. A seleção das escolas foi feita por meio de amostragem por área (Cozby, 1981). Inicialmente, foi feito o contato com as escolas para apresentação do projeto de pesquisa e da documentação necessária. Obtida a aprovação do projeto pela equipe técnica da escola e pelos professores, foram encaminhados para os pais ou responsáveis os termos de consentimento. Este estudo esteve de acordo com o decreto $n^{\circ}$ 93.933/1988 do Conselho Nacional de Saúde em seu capítulo II 4 e 5 e com a Resolução $n^{\circ}$ 016/2000 do Conselho Federal de Psicologia, que versam sobre os aspectos éticos da pesquisa com seres humanos. Salvaguardou-se a todos os participantes o direito de sigilo, voluntariado e interrupção da participação. A explicação do estudo, seus objetivos e finalidades foram apresentados de forma coletiva para as crianças, na própria sala de aula.

Testagem da versão preliminar da escala (com 60 itens)

Após os termos terem sido assinados pelos pais e/ou responsáveis, a escala foi aplicada coletivamente na sala de aula. Durante a zplicação, a presença dos professores foi facultativa. A aplicação foi realizada pelo pesquisador coordenador e porum auxiliar de pesquisa.

As crianças receberam as seguintes instruções, que eram acompanhadas pelo desenho da escala de 
respostas no quadro-negro da sala, com o objetivo de instruí-las sobre o preenchimento e utilização corretos da escala tipo Likert do instrumento:

"Aseguirvocêstêm uma lista defrasesqueindicam o que crianças e pré-adolescentes pensam sobre vários assuntos. Vocêsdevem lercada fraseemarcaro quanto vocês concordam com o que ela diz. Sevocês concordarem nem um pouco, marquem (1); se concordarem um pouco, marquem (2); se concordarem mais ou menos, marquem (3); se concordarem bastante, marquem (4) e se concordarem muitíssimo, marquem (5). Vocês devem responder individualmente. Não há respostascertasnem erradas, vale o quevocês pensam. Setiverem dúvidas, levantem o braço eserão atendidasna classe."

\section{Participantes}

Participaram do estudo 1, 661 crianças de ambosossexos, 345 meninos (52,2\%)e 316 meninas (47,8\%). A faixa etária variou entre 7 e 12 anos (média - $M=10,6$ anos; desvio-padrão - DP=1,7 anos). As crianças freqüentavam o ensino fundamental, entre a $2^{\underline{a}}$ e a $6^{\underline{a}}$ séries, de escolas públicas estaduais $(54,0 \%)$ e privadas $(46,0 \%)$ de Porto Alegre. Desta amostra, $66,0 \%$ das crianças moravam com ambos os pais, $31,0 \%$ viviam com somente um dos pais e 3,0\% não viviam com esses. 0 número médio de irmãos relatados pelas crianças foi de 1,5 irmãos ( $D P=1,3)$.

Resultados do estudo 1

Recorrendo-se a análises fatoriais exploratórias, e delimitando-se como critério de extração todo o fator com Eigenvalue maior que 1 (Kaiser, 1960), buscou-se verificar quais itens compunham fatores relacionados aos domínios de satisfação de vida infantil. Apesar de se ter esses itens já elaborados, visto que os mesmos partiam do modelo teórico proposto, foi necessária a realização de análises exploratórias, uma vez que tal modelo nunca tinha sido testado antes. Considerou-se mais adequada a adoção da rotação Direct Oblimin, específica para extração de fatores correlacionados, pois, conforme estudos anteriores, os fatores de domínios de satisfação de vida representam construtos correlacionados (Huebner, 1994a). A partir desse procedimento, foram extraídas 13 dimensões da escala, explicando
53,83\% da variância. O Scree Plot (Cattell, 1966) indicou que uma solução de seisfatores seria adequada. Análises forçando a extração de três a seis fatores confirmaram que seis fatores constituíam a melhor solução. Os fatores foram denominados de Self, Self Comparado, Não-Violência, Família, Amizade e Escola.

Os fatores extraídos confirmaram alguns dos domínios apresentados pelo Modelo Multidimensional de Satisfação de Vida Infantil de Huebner (self, família, amizade e escola). Verificou-se a permanência dos itens relativos ao domínio "não-violência". Observou-se o surgimento de um novo fator, denominado selfcomparado. Os fato res ficaram caracterizados:

- Self: este fator é composto por itens que descrevem o self como positivo, com características positivas, como auto-estima, bom-humor, capacidade de relacionar-se, capacidade de demonstrar afeto etc.;

- Self Comparado: este fator agrupa itens que se caracterizam por realizar avaliações comparativas com seus pares. Os itens possuem conteúdos relacionados ao lazer, à amizade e à satisfação de desejos e afetos.

- Não-violência: este fator inclui itens que possuem conteúdos associados a comportamentos agressivos;

- Família: envolve itens descritores de um ambiente familiar saudável, harmônico, afetivo, de relacionamentos satisfatórios, além de indicações de satisfação quanto à diversão;

- Amizade: fator que se caracteriza pelos relacionamentos com pares, nível de satisfação desses relacionamentos e algumas indicações ao lazer, situações de diversão e apoio;

- Escola: os itens descrevem a importância da escola, do ambiente escolar, dos relacionamentos interpessoais nesse espaço e o nível de satisfação em relação a esse ambiente.

Essa estrutura fatorial encontrada tem sentido teórico e, segundo Pasquali (1999), a partir dessa análise, pode-se considerar que 0 instrumento apresenta validade de construto. Os itens que não apresentaram cargas superiores a $0,30 \mathrm{em}$ nenhum fator foram eliminados. Os itens com cargas superiores a 0,30 em mais de um fator foram mantidos na escala final, em 
função de sua contribuição teórica para a dimensão. A Escala Multidimensional finalizou com 50 itens, distribuídos em seis fatores, explicando $46,5 \%$ da variância total. Na Tabela 1, é apresentada a Matriz Fatorial dos Itens da Escala Multidimensional de Satisfação de Vida Infantil, composta pelos itens que permaneceram agrupados pelas dimensões às quais pertencem, em ordem decrescente de suas cargas fatoriais, bem como seus Eigenvalues e os valores da variância explicada.

O número de itens, a média, 0 desvio padrão de cada dimensão e da escala total, bem como seusíndices de fidedignidade (Alfa de Cronbach) são apresentados na Tabela 2. Cabe ressaltar que a média foi calculada pelo somatório total dividido pelo número de itens, uma vez que cada sub-escala apresenta diferenças na quantidade dos itens que a compõem.

As sub-escalas apresentaram índices de fidedignidade satisfatórios, variando entre 0,82 e 0,86, com exceção da sub-escala não-violência, que apresentou um valor de 0,66 . Pode-se justificar esse valor mais baixo devido ao pequeno número de itens dessa sub-escala $(n=4)$. O Alpha obtido para a escala total foi elevado: 0,93 . Foram encontradas correlações significativas entre as sub-escalas, conforme se pode observar na Tabela 3 , que apresenta os valores de correlação de Pearson entre as seis sub-escalas.

Para investigar os efeitos do sexo, do tipo de escola e da faixa etária das crianças, foi realizada uma Análise de Variância Multivariada (MANOVA) 2×2×3, tendo como variáveis dependentes as seis sub-escalas avaliadas acima. Não foram encontrados efeitos de interação $\mathbb{F}(2,606)>1]$. Foram identificados efeitos principais para tipo de escola e faixa etária em algumas das sub-escalas da EMSVC. Quanto ao tipo de escola, foram verificados efeitos principais significativos nas sub-escalas: Self $[F(1,606)=5,48 ; p<0,02]$, SelfComparado $[F(1,606)=22,54 ; p<0,01]$, Não-Violência $F(1,606)=12,57$; $p<0,01]$, Família $F(1,606)=14,41 ; p<0,01]$ e Amizade $[F(1,606)=16,40 ; p<0,01]$.

Crianças de escolas públicas reportaram menores níveis de satisfação do que crianças de escolas privadas em todos os domínios de satisfação de vida infantil, com exceção da escola, na qual não foram
Self [pública ( $M=4,08 ; D P=0,64) ;$ privada $(M=4,18$; $\mathrm{DP}=0,56)$ ], Self Comparado [pública ( $M=3,48 ; \mathrm{DP}=0,91$ ); privada ( $M=3,91 ; D P=0,77)$ ], Família [pública $(M=4,48$; $D P=0,56)$; privada $(M=4,64 ; D P=0,39)$ ], Não-Violência [pública ( $M=4,09 ; \mathrm{DP}=0,88)$; privada $(\mathrm{M}=4,34 ; \mathrm{DP}=0,69)$ ] e Amizade [pública $(M=4,11 ; D P=0,65)$; privada $(M=4,30$; $\mathrm{DP}=0,59)]$.

Foram encontradas diferenças significativas de idade nas sub-escalas: Self $[F(2,606)=7,14 ; p<0,01]$, Self Comparado $[F(2,606)=2,93 ; p<0,05]$, Família $[F(2,606)=6,89$; $p<0,01]$, Amizade $[F(2,606)=5,10 ; p<0,01]$ e Escola $[F(2,606)=34,03 ; p<0,01]$. 0 Teste para Comparação de Médias a posteriori (Tukey) apontou para diferenças significativas entre as faixas etárias para cada sub-escala. No domínio Self, crianças de 7-8 anos ( $M=4,29 ; D P=0,61$; $p=0,02$ ) relataram maiores níveis de satisfação consigo do que as crianças de $11-12$ anos ( $M=4,05 ; D P=0,60)$. No domínio SelfComparado, foram observadas diferenças significativas entre as crianças de 7-8 anos ( $M=3,37$; $D P=0,98)$, as crianças de $9-10$ anos $(M=3,72 ; D P=0,86)$ e as crianças de $11-12$ anos ( $M=3,76 ; D P=0,84)$. Quanto à Família, observou-se que as crianças mais velhas, 11-12 anos $(M=4,49 ; D P=0,55)$ apresentaram menores níveis de satisfação do que as crianças de 9-10 anos ( $M=4,63$; $D P=0,41)$. Crianças da segunda faixa etária, 9-10 anos $(M=4,30 ; D P=0,58)$, apresentaram maiores níveis de satisfação do que crianças mais velhas, da terceira faixa etária $(M=4,12 ; D P=0,66)$, no que se refere à Amizade. Quanto à Escola, observou-se que as crianças entre $7 \mathrm{e}$ 8 anos ( $M=4,55 ; D P=0,58$ ) apresentaram maiores níveis de satisfação do que crianças de 9 e 10 anos ( $M=4,40$; $\mathrm{DP}=0,56)$ e crianças de $11-12$ anos $(\mathrm{M}=4,04 ; \mathrm{DP}=0,66)$. Não foram constatadas diferenças significativas entre os sexos em nenhum dos domínios relativos à satisfação de vida infantil.

Estudo 2: estudo de validação concorrente da EMSVC

\section{Procedimentos}

Escolha dos instrumentos para comparação com os resultados obtidos com a EMSVC

Após a obtenção da fidedignidade do instrumento, são necessários, para dar continuidade ao 
Tabela 1. Matriz fatorial dos itens da escala multidimensional de satisfação de vida infantil.

\begin{tabular}{|c|c|c|c|c|c|c|c|}
\hline $\mathrm{n}$ & Sentença & 1 & 2 & 3 & 4 & 5 & 6 \\
\hline \multicolumn{8}{|c|}{ Self } \\
\hline 1 & Eu sou uma pessoa bem humorada. & 0,60 & & & & & \\
\hline 5 & Eu so rrio bastante. & 0,60 & & & & & \\
\hline 12 & Eu sou divertido. & 0,59 & & & & & \\
\hline 29 & Eu sou alegre. & 0,51 & & & & & \\
\hline 50 & Mantenho a calma. & 0,51 & & 0,36 & & & \\
\hline 10 & Eu me sinto calmo, tranqüilo. & 0,49 & & & & & \\
\hline 45 & Eu sou uma pessoa carinhosa. & 0,41 & & & & & \\
\hline 56 & Tenho facilidade para fazer amigos. & 0,37 & & & & & \\
\hline 42 & Eu sou esperto. & 0,36 & & & & & \\
\hline 38 & Eu me divirto com muitas coisas. & 0,31 & & & & & \\
\hline \multicolumn{8}{|c|}{ Self comparado } \\
\hline 26 & Meus amigos podem fazer mais coisas do que eu. & & 0,81 & & & & \\
\hline 37 & Meus amigos se divertem mais do que eu. & & 0,77 & & & & \\
\hline 9 & Meus amigos são mais alegres do que eu. & & 0,76 & & & & \\
\hline 63 & Meus amigos brincam mais do que eu. & & 0,71 & & & & \\
\hline 14 & Meus amigos ganham mais presentes do que eu. & & 0,70 & & & & \\
\hline 3 & As outras crianças são mais alegres do que eu. & & 0,68 & & & & \\
\hline 67 & As outras crianças têm mais amigos do que eu. & & 0,65 & & & & \\
\hline 46 & Preciso receber mais atenção. & & 0,30 & & & & \\
\hline \multicolumn{8}{|c|}{ Não-violência } \\
\hline 33 & Gosto de brigas. & & & 0,69 & & & \\
\hline 16 & Brigo muito com meus amigos. & & & 0,65 & & & \\
\hline 64 & Sou imitado. & 0,35 & & 0,60 & & & \\
\hline 60 & Brigar resolve os problemas. & & & 0,56 & & & \\
\hline \multicolumn{8}{|c|}{ Família } \\
\hline 27 & Meus pais são carinhosos comigo. & & & & 0,75 & & \\
\hline 2 & Minha família gosta de mim. & & & & 0,66 & & \\
\hline 43 & Eu me divirto com a minha família. & & & & 0,65 & & \\
\hline 11 & Minha família me faz feliz. & & & & 0,61 & & \\
\hline 61 & Gostaria que minha família fosse diferente. & & & & 0,59 & & \\
\hline 24 & Os membros da minha família se dão bem. & & & & 0,55 & & \\
\hline 30 & Minha família me ajuda quando preciso. & & & & 0,54 & & \\
\hline 47 & Eu fico feliz quando a minha família se reúne. & & & & 0,45 & & \\
\hline 20 & Eu me divirto com as coisas que eu tenho. & & & & 0,44 & & \\
\hline 34 & Tenho pessoas que me ajudam. & & & & 0,42 & & \\
\hline 15 & Procuro fazer coisas que me deixam feliz. & & & & 0,32 & & \\
\hline \multicolumn{8}{|c|}{ Amizade } \\
\hline 49 & Estou satisfeito com os amigos que tenho. & & & & & $-0,62$ & \\
\hline 25 & Meus amigos me ajudam quando eu preciso. & & & & & $-0,57$ & \\
\hline 58 & Meus amigos gostam de mim. & & & & & $-0,56$ & $-0,30$ \\
\hline 31 & Eu gostaria que meus amigos fossem diferentes. & & & & & $-0,52$ & \\
\hline 54 & Eu me divirto com meus amigos. & 0,32 & & & & $-0,51$ & \\
\hline 68 & Gosto de conversar com meus amigos. & & & & & $-0,46$ & \\
\hline 48 & Meus amigos brigam muito comigo. & & & 0,40 & & $-0,40$ & \\
\hline 35 & Eu me relaciono bem com meus colegas. & 0,33 & & & & $-0,38$ & \\
\hline 41 & É bom brincar com meus amigos. & & & & & $-0,37$ & \\
\hline 32 & Sempre encontro ajuda quando preciso. & & & & & $-0,33$ & \\
\hline \multicolumn{8}{|c|}{ Escola } \\
\hline 36 & Eu gosto de ir à escola. & & & & & & $-0,81$ \\
\hline 62 & Eu gosto das atividades da escola. & & & & & & $-0,76$ \\
\hline 53 & Meus professores gostam de mim. & & & & & & $-0,65$ \\
\hline 65 & Eu aprendo muitas coisas na escola. & & & & & & $-0,61$ \\
\hline 57 & Eu me sinto bem na minha escola. & & & & & $-0,35$ & $-0,57$ \\
\hline 51 & Eu me divirto na escola. & & & & & $-0,36$ & $-0,48$ \\
\hline \multirow[t]{3}{*}{44} & Eu gosto de ajudar as pessoas. & & & & & & $-0,39$ \\
\hline & Eigenvalues & 14,16 & 3,94 & 2,41 & 1,91 & 1,76 & 1,65 \\
\hline & \% Variância Explicada & 24,25 & 7,31 & 4,65 & 3,73 & 3,35 & 3,18 \\
\hline
\end{tabular}


Tabela 2. Propriedades das dimensões e da escala total multidimensional de satisfação de vida infantil.

\begin{tabular}{lcccc}
\hline Propriedade & Itens $(\mathrm{n})$ & Média & DP & Alfa de Cronbach \\
\hline 1. Self & 10 & 4,19 & 0,60 & 0,82 \\
2. Self comparado & 8 & 3,69 & 0,88 & 0,86 \\
3. Não-violência & 4 & 4,21 & 0,81 & 0,66 \\
4. Família & 11 & 4,57 & 0,50 & 0,82 \\
5. Amizade & 10 & 4,21 & 0,63 & 0,82 \\
6. Escola & 7 & 4,23 & 0,68 & 0,83 \\
\hline Escala total & 50 & - & - & 0,93 \\
\hline
\end{tabular}

DP: desvio-padrão.

Tabela 3. Matriz de correlação entre os fatores (r).

\begin{tabular}{lcccccc}
\hline & 1 & 2 & 3 & 4 & 5 & 6 \\
\hline 1. Self & & & & & & \\
2. Self comparado & $0,32^{* *}$ & & & & & \\
3. Não-violência & $0,25^{* *}$ & $0,35^{* *}$ & & & & \\
4. Família & $0,63^{* *}$ & $0,40^{* *}$ & $0,22^{* *}$ & & & \\
5. Amizade & $0,61^{* *}$ & $0,42^{* *}$ & $0,37 *$ & $0,60^{* *}$ & & \\
6. Escola & $0,54 *$ & $0,22^{* *}$ & $0,27 * *$ & $0,51 * *$ & $0,56 * *$ & - \\
\hline
\end{tabular}

$* * p \leq 0,01$.

desenvolvimento do mesmo, os processos de validação. Uma das formas usualmente utilizadas para se verificar a validade de um instrumento psicológico é demonstrando que ele possui resultados compatíveis aos de outros instrumentos que avaliam os mesmos construtos ou construtos correlatos (validação concorrente). Como, no Brasil, não existem instrumentos construídos e validados, ou adaptados para avaliar a satisfação de vida infantil, com exceção da Escala de Satisfação de Vida de Estudantes de Huebner, já adaptada para o português por Giacomoni (1998), cujas características psicométricas foram insatisfatórias, optou-se por utilizar instrumentos que avaliassem construtos correlatos ao bem-estar subjetivo.

Assim, para avaliar as variáveis correlatas, foram selecionados instrumentos que já foram utilizados no Brasil ou que tiveram seus processos de adaptação e/ou normatização realizados. As variáveis eleitas foram: auto-estima, ansiedade e depressão, tendo em vista que, em alguns estudos, apresentaram correlações significativas com o bem-estar subjetivo (Huebner, 1991b; Laurent et al. 1999). Além dessas variáveis, também foi investigada a satisfação de vida global de crianças.
Para avaliar auto-estima, foi utilizada a versão adaptada para o português (Hutz, 2000) da Escala de Auto-Estima de Rosenberg (1965). Esse instrumento é uma escala de auto-relato do tipo Likert (quatro pontos), composta originalmente por dez itens, que investigam aspectos globais da auto-estima. A escala foi desenvolvida para adolescentes, sendo bastante difundida devido à sua praticidade; entretanto, sua utilização em crianças a partir de oito anos de idade tem sido comum (Houtte \& Jarvis, 1995). A versão adaptada da escala adicionou mais um item, mantendo-se a estrutura unidimensional da mesma (Hutz, 2000). Os participantes devem indicar o grau de concordância com 0 item descrito. Quanto maior o escore obtido, maior a auto-estima. A escala tem apresentado índices de fidedignidade constantes e aceitáveis para uso em pesquisa (acima de 0,80). A pesquisa realizada com a escala adaptada demonstrou parâmetros psicométricos apropriados (Hutz, 2000). Neste estudo de validação da EMSVC, a Escala de Auto-Estima apresentou um índice de consistência interna de 0,75 (Alpha de Cronbach).

$A$ ansiedade foi medida utilizando-se o Inventário de Ansiedade Traço-Estado (IDATE-C),já adaptado e padronizado para o seu uso no Brasil por Biaggio (1980). O IDATE-C foi desenvolvido a partir do Inventánio de Ansiedade Traço-Estado (Spielberger, 1983) na forma adulta (Spielberger, Gorsuch \& Lushene, 1979; Biaggio, Natalício \&Spielberger, 1977).A forma infantil écomposta por duas escalas do tipo auto-relato, que visam medir a ansiedade-estado (FORMA C-1) e a ansiedade-traço (FORMA C-2), dois conceitos distintos de ansiedade. É utilizada com crianças de Ensino Fundamental, e é composta por 20 itens em cada escala, podendo ser aplicada de forma coletiva. Os valores encontrados, neste estudo, quanto à fidedignidade, foram satisfatórios. Para a forma C-1 foi encontrado um Alpha de Cronbach de 0,84, e para a forma C-2, Alpha de Cronbach de 0,73.

Para avaliar a depressão, foi utilizado o Children's Depression Inventory (CDI), elaborado por Kovacs (1983, 1992), a partir do Beck Depression Inventory para adultos. 0 objetivo do CDI é detectar a presença e a severidade do transtorno depressivo na infância. Destina-se a identificar alterações afetivas em crianças e adolescentes dos sete aos dezessete anos. É uma medida unifatorial composta por 27 itens, cada um constando de três 
opções de resposta (pontuada como 0,1 ou 2), na qual a criança deve assinalar a que melhor descreve 0 seu estado nos últimos tempos. O CDI pode ser aplicado tanto individualmente, quanto coletivamente. Em relação às propriedades psicométricas da escala, a consistência interna descrita por Kovacs (1980/1981) mostrou-se adequada $(0,86)$. Kovacs estabeleceu como ponto de corte do CDI o escore 19. No Brasil, o CDI foi adaptado por Hutz e Giacomoni (2000). As pesquisas que utilizaram versões adaptadas para o contexto brasileiro vêm apresentando condições psicométricas adequadas. 0 Alpha de Cronbach do inventánio adaptado para o uso no nordeste do país por Gouveia, Barbosa, Almeida e Gaião (1995) foi de 0,81. Em pesquisas realizadas com amostras infantis no Rio Grande do Sul, observaram-se os seguintes coeficientes de Alpha de Cronbach:0,80 (Giacomoni, 1998) e 0,92 (Reppold, 2001). Outros estudos que avaliaram crianças e adolescentes gaúchos em situação de risco obtiveram um Alpha de até 0,79 (Dell'Aglio, 2000; Silva, 2001). Neste estudo, foi encontrado um Alpha de Cronbach de 0,81.

A satisfação de vida global das crianças foi avaliada pela Escala de Satisfação de Vida Global Infantil (Giacomoni, 2002). Esta é uma medida curta (7 itens), de fácil aplicação e econômica, indicada para avaliar a satisfação de vida global em crianças a partir de sete anos de idade. A escala, de tipo Likert de cinco pontos, apresenta uma estrutura fatorial unidimensional, consistência interna adequada e correlações apropriadas com outras medidas. 0 Alpha de Cronbach obtido para a escala de sete itens foi de 0,83 . Como cada item podia ter escores de resposta variando de um a cinco, a amplitude da escala, portanto, varia de 5 (baixa satisfação) a 35 (alta satisfação).

Seleção das escolas e aplicação dos instrumentos

Seguiu-se os mesmos procedimentos e cuidados éticos já descritos no Estudo 1. As escolas foram contatadas para a apresentação do projeto e para o encaminhamento dos termos de consentimento informado aos pais e/ou responsáveis. As escalas foram aplicadas de forma coletiva, em sala de aula, durante somente um encontro. A ordem de aplicação dos instrumentos foi aleatória.
Participantes

Para a realização do estudo de validação concorrente, participaram 230 crianças de ambos os sexos, 117 meninos (50,90\%) e 113 meninas (49,18\%). A faixa etária variou entre oito e 12 anos ( $M=10,6$ anos; $D P=1,7$ anos). As crianças freqüentavam o Ensino Fundamental (de 3 a a 6 a série) de escolas públicas estaduais $(57,40 \%$ ) e privadas $(42,60 \%)$ de Porto Alegre. Os dados demográficos da amostra são descritos na Tabela 4.

\section{Resultados do Estudo 2}

Para a verificação da validade concorrente da ESVMC foram examinados os padrões de correlações entre si, assim como entre esses e os da Escala de Auto-Estima, do Inventário de Depressão Infantil (CDI) e das Escalas do Inventário de Ansiedade Traço-Estado (IDATE-C, FORMA C1-Estado e FORMA C2-Traço). A Tabela 5 apresenta as correlações entre seus fatorese os demais instrumentos eleitos, inclusive a Escala de Satisfação de Vida Global.

As correlações obtidas entre a Escala de Satisfação de Vida Global e os instrumentos que avaliam auto-estima ( $r=0,51, p<0,01)$, depressão $(r=-0,52, p<0,01)$, ansiedade-traço $(r=-0,32, p \leq 0,01)$ e ansiedade-estado $(r=-0,52, p<0,01)$ ocorreram no sentido esperado, predito,

Tabela 4. Dados demográficos da amostra.

\begin{tabular}{lcc}
\hline Variável & $\mathrm{n}$ & $\%$ \\
\hline Sexo & 113 & 49,1 \\
Feminino & 117 & 50,9 \\
Masculino & & \\
Faixa etária & 103 & 44,8 \\
8-9-10 anos & 127 & 55,2 \\
11-12 anos & & \\
Tipo de escola & 132 & 57,4 \\
Pública & 98 & 42,6 \\
Privada & & \\
Série & 36 & 15,7 \\
3a Série & 69 & 30,0 \\
4a Série & 51 & 22,2 \\
5a Série & 74 & 32,2 \\
6a Série & 230 & 100,0 \\
\hline Total & & \\
\hline
\end{tabular}


Tabela 5. Matriz de correlação entre assub-escalas de satisfação de vidamultidimensional ea escala de satisfação de vida global e os escores de auto-estima, depressão, ansiedade-traço e ansiedade-estado.

\begin{tabular}{|c|c|c|c|c|c|c|c|c|c|c|}
\hline \multirow{2}{*}{ Sub-escalas } & \multicolumn{2}{|c|}{ Satisfação global de vida } & \multicolumn{2}{|c|}{ Auto-estima } & \multicolumn{2}{|c|}{ CDI } & \multicolumn{2}{|c|}{ IDATE C1 estado } & \multicolumn{2}{|c|}{ IDATE C2 traço } \\
\hline & $p$ & $\mathrm{n}$ & $p$ & $\mathrm{n}$ & $p$ & $n$ & $p$ & $n$ & $p$ & $n$ \\
\hline 1. Self & 0,70 & 648 & 0,47 & 227 & $-0,43$ & 214 & $-0,51$ & 222 & $-0,30$ & 215 \\
\hline 2. Self comparado & 0,38 & 640 & 0,49 & 223 & $-0,36$ & 210 & $-0,29$ & 218 & $-0,28$ & 211 \\
\hline 3. Não-violência & 0,23 & 645 & 0,37 & 229 & $-0,42$ & 216 & $-0,22$ & 224 & $-0,16$ & 214 \\
\hline 4. Família & 0,75 & 649 & 0,47 & 227 & $-0,51$ & 214 & $-0,41$ & 222 & $-0,33$ & 215 \\
\hline 5. Amizade & 0,59 & 643 & 0,45 & 229 & $-0,43$ & 216 & $-0,44$ & 224 & $-0,21$ & 217 \\
\hline 6. Escola & 0,53 & 650 & 0,43 & 229 & $-0,54$ & 216 & $-0,33$ & 224 & $-0,21$ & 217 \\
\hline Satisfação global de vida & & & 0,51 & 228 & $-0,52$ & 215 & $-0,52$ & 223 & $-0,32$ & 216 \\
\hline
\end{tabular}

Todas as correlações são significativas $(p<0,01)$.

e em magnitudes coerentes com a literatura. Huebner (1991b) encontrou uma correlação de 0,65 $(p<0,01)$ entre a sua escala unidimensional de satisfação de vidaglobal e auto-estima infantil (escala de Rosenberg). Nesse mesmo estudo, encontrou uma correlação de $-0,51$ $(p=0,01)$ entre a satisfação e ansiedade geral infantil (CDI). Em outro estudo, Huebner e Aldermam (1993) encontraram uma correlação entre depressão e o nível de satisfação de vida global $(r=-0,57, p<0,01)$ similar à encontrada neste estudo de validação $(r=-0,52, p<0,01)$.

Observa-se uma alta correlação entre a Escala de Satisfação de Vida Global e os domínios relativos à Família $(r=0,75, p<0,01)$ e ao Self $(r=0,70, p<0,01)$. Esse resultado referente à Família, assim como a mais alta correlação entre todos os domínios investigados e a satisfação de vida global, vai ao encontro do estudo desenvolvido por Huebner (1991b), no qual a família, e não a amizade, apareceu mais fortemente associada à satisfação global. Terry e Huebner (1995) também confirmaram, em outro trabalho, o resultado referente aos domínios relativos aos relacionamentos interpessoais íntimos como os preditores mais fortes da satisfação de vida global. As correlações positivas encontradas entre a Escala de Satisfação de Vida Global e os domínios Amizade $(r=0,59, p<0,01)$ e Escola $(r=0,53, p<0,01)$ também são consideradas satisfatórias.

A Escala de Auto-estima de Rosenberg apresentou correlações positivas de magnitudes modestas (variando de 0,37 a 0,49) com todos os domínios de satisfação de vida infantil. Contudo, chama a atenção que o domínio Self Comparado foi o domínio que apresentou a maior correlação $(=0,49)$. Tal resultado apresenta mais evidências da importância das comparações sociais entre as crianças.
O Inventário de Depressão Infantil correlacionou-senegativamente com todos os domínios de satisfação de vida infantil, reforçando evidências de validade concorrente da EMSVC. O mesmo vale para as Escalas de Ansiedade Traço e Estado.

\section{Discussão}

O objetivo deste estudo foi desenvolver uma escala, intitulada Escala Multidimensional de Satisfação de Vida para Crianças (EMSVC), assim como apresentar informações substanciais so bre as propriedades psicométricas da mesma. Paralelamente, viabilizou-se também a testagem do Modelo Multidimensional de Satisfação de Vida Infantil de Huebner. As análises iniciais desta escala de auto-relato infantil identificaram fatores coerentes, que representam domínios de satisfação de vida infantil, diferenciados por crianças a partir de sete anos de idade.

Verificou-se que a estrutura fatorial da escala envolve dimensões psicologicamente significativas. Os fatores extraídos: Self, Self Comparado, Não-Violência, Família, Amizadee Escola possuem sentido teórico, uma vezque confirmam os domínios propostos por Huebner (1994a). Foram encontradas consistências internas adequadas para cada uma das sub-escalas e para a escala total, com exceção da sub-escala Não-Violência, que possui indicações para que seja aprimorada e que tenha seu número de itens aumentado.

A validade concorrente das sub-escalas da EMSVC foi confirmada por correlações médias com medidas critério. Os achados referentes às variáveis sócio-demográficas: sexo, idade e tipo de escola são 
coerentes com a literatura. Novamente, não foram verificadas diferenças entre os sexos quanto aos níveis de satisfação de vida com os domínios. Este resultado acrescenta mais evidências no que diz respeito à ausência de diferenças entre gêneros quanto à satisfação de vida em diferentes culturas.

No entanto, os resultados relativos a possíveis diferenças entre faixas etárias discordam dos de Huebner (1994a), que não as encontrou. Nos domíniosSelf,Família, Amizade e Escola, as crianças mais novas apresentaram níveis de satisfação superiores aos das crianças mais velhas. Pode-se observar um decréscimo de satisfação com o passar dos anos. Contudo, no domínio Self Comparado as crianças mais velhas ap resentaram níveis mais elevados de satisfação do que as crianças menores. Tal resultado merece uma investigação mais específica sobre as razões de tal processo.

Quanto às diferenças entre crianças de escola pública e privada, os resultados são consistentes em apontar que crianças de escolas públicas apresentam menores níveis de satisfação de vida nos domínios Self, Self Comparado, Não-Violência, Família, Amizade. Este achado pode corroborar a premissa de que crianças de escolas públicas sofrem de piores condições de vida, se comparadas às crianças de escolas privadas. As causas destas diferenças e o possível impacto da variável nível sócio-econômico precisam ser melhor exploradas.

As análises realizadas neste estudo indicam que a escala construída apresenta qualidades psicométricas adequadas, com a extração de fatores que possuem sentido teórico, e apresentam, igualmente, uma alta consistência interna, confirmando um sentido teórico de avaliação da satisfação de vida infantil. Não podemos deixar de salientar ressalvas quanto à limitação da amostra, uma vez que essa não possui qualidades suficientes para se tornar capaz de representar a população brasileira. 0 estudo realizado deveria ser ampliado para amostras em todas as regiões do país, e para diferentes classes sócio-econômicas e culturais.

Respeitando e seguindo as orientações e parâmetros para Testes Educacionais e Psicológicos (American Psychological Association, 1985), não se pode limitar a qualidade de qualquer instrumento de avaliação apenas às evidências de precisão. As evidências de validade são também fundamentais para confirmar a efetividade do teste. Faz-se ainda necessário investigar se a escala possui poder discriminante entre grupos com características diferenciadas (validade de critério).

O desenvolvimento da EMSVC, juntamentecom outras medidas que avaliem o afeto, viabiliza a avaliação de bem-estar subjetivo infantil na realidade brasileira. Primeiramente, os instrumentos aqui apresentados fornecem evidências de que o bem-estar infantil pode ser acessado de forma confiável e válida em crianças a partir de sete anos de idade. Em segundo lugar, o desenvolvimento de instrumentos de bem-estar possibilita a realização de pesquisas programadas sobre a natureza e o desenvolvimento de saúde mental positiva como, por exemplo, felicidade e satisfação de vida em crianças e jovens. Por fim, essas medidas permitirão a exploração de diferenças no bem-estar subjetivo de crianças de diferentes culturas, quanto aos domínios específicos da vida dessas.

\section{Referências}

Adelman, H. S., Taylor, L., \& Nelson, P. (1989). Minors' dissatisfaction with their life circumstances. Child Psychiatry and Human Development, 20 (2), 135-147.

American Psychological Association. (1985). Standards for educational and psychological testing. Washington, DC: Author.

Andrews, F. M., \& Whitey, S. B. (1976). Social indicators of well-being:America's perception of quality of life. New York: Plenum Press.

Assump ção, F. B., Kuczynski, E., Sprovieri, M. H., \& Aranha, E. M. G. (2000). Escala de avaliação de qualidade de vida (AUQEI - autoquestionnaire qualité de vie enfant imagé): validade e confiabilidade de uma escala para qualidade de vida em crianças de 4 a 12 anos. Arquivos de Neuropsiquiatria, 58 (1), 119-127.

Biaggio, A. M. B. (1980). Desenvolvimento da forma infantil em português do inventário de ansiedade traço-estado de Spielberger. ArquivosBrasileirosdePsicologia Aplicada, 32 (3), 106-118.

Biaggio, A. M. B., Natalício, L., \& Spielberger, C. D. (1977). Desenvolvimento da forma experimental em português do inventário de ansiedade traço-estado (IDATE) de Sp ielberger. ArquivosBrasileiros dePsicologia Aplicada, 29 (3): 31-44.

Campbell, A., Converse, P., \& Rodgers, W. L. (1976). The quality of American life: perceptions, evaluations, and satisfactions. New York: Russell Sage Foundation.

Cattell, R. B. (1966). The meaning and strategic use of factor analysis. In R. B. Cattell (Org.), Handbook of multivariate experimental psychology (pp.174-243). Chicago: Rand McNally. 
Cozby, P. (1981). Methods in behavioral research. California: Mayfield Publishing.

Dell'Aglio, D. D. (2000). O processo de coping, institucionalização e eventos de vida em crianças e adolescentes. Tese de doutorado não-pub licada, UniversidadeFederal do Rio Grande do Sul, Porto Alegre.

Diener, E. (1984). Subjective well-being. Psychological Bulletin, 95 (3), 542-575.

Diener, E. (1994). Assessing subjective well-being:progress and opportunities. Social Indicators Research, 31 (2), 103-157.

Diener, E., Emmons, R., Larsen, R., \& Griffin, S. (1985). The satisfaction with life scale.J ournal ofPersonalityAssessment, 49 (1), 91-95.

Evans, D. R., Burns, J. E., Robinson, W. E., \& Garret, D. (1985). The quality of life questionnaire: a multidimensional measure. American J ournal of Community Psychology, 13 (3), 305-322.

Frisch, M. B., Cornell, J., Villanueva, M., \& Retzlaff, P. J. (1992). Clinical validation of the quality of life inventory: a measure of life satisfaction for use in treatment planning and outcome assessment. Psychological Assessment, 4 (1), 92-101.

Giacomoni, C. H. (1998). Desempenho escolar, controle percebido e eventos de vida como preditores de bem-estar subjetivo em crianças. Dissertação de mestrad o não- publicada, Universidade Federal do Rio Grande do Sul, Porto Alegre.

Giacomoni, C. H. (2002). Bem-estar subjetivo infantil:conceito de felicidade e construção de instrumentos para avaliação. Tese de doutorado não-publicada, Universidade Federal do Rio Grande do Sul, Porto Alegre.

Gilman, R., Huebner, E. S., \& Laughlin, J. E. (2000). A first study of the multidimensional students' life satisfaction scale. Social Indicators Research, 52 (2), 135-160.

Gouveia, V. V., Barbosa, G. A., Almeida, H. J. F., \& Gaião, A. A. (1995). Inventário de depressão infantil - CDI: estudo de adaptação com escolares de J oão Pessoa.J ornal Brasileiro de Psiquiatria, 44 (7), 345-349.

Greespoon, P. J., \& Saklofske, D. H. (1998). Confirmatory factor analysis of the multidimensional students' life satisfaction scale. Personality and Individual Differences, 25 (5), 965-971.

Harding, L. (2001). Children's quality of life assessments: a review of generic and health related quality of life measures completed by children and adolescents. Clinical Psychology and Psychotherapy, 8 (2), 79-86.

Headey, B., Holmstrom, E., \&Wearing, A. (1984). The impact of life events and changes in domain satisfactions on well-being. Social Indicators Research, 15 (3), 203-227.

Houtte, B. A., \& Jarvis, P. A. (1995). The role of pets in preadolescent psychological development. Journal of Applied Developmental Psychology, 16 (3), 463-479.

Huebner, E. S. (1998a). Cross-racial application of a children's multidimensional life satisfaction scale.SchoolPsychology International, 19 (2), 179-188.
Huebner, E. S. (1998b). Cross-racial ap plication of a children's multidimensional life satisfaction scale: erratum. School Psychology International, 19, (4), 290.

Huebner, E. S. (1995). The students' life satisfaction scale: an assessment of psychometric properties with black and white elementary school students. Social Indicators Research, 34 (2), 315-323.

Huebner, E. S. (1994a). Preliminary development and validation of a multidimensional life satisfaction scale for children. Psychological Assessment, 6 (2), 149-158.

Huebner, E. S. (1994b). Conjoint analyses of the students' life satisfaction scale and the Piers-Harris self-concept scale. Psychology in theSchools, 31 (4), 273-277.

Huebner, E. S. (1991a). Initial development of the student's life satisfaction scale. School Psychology International, 12 (3), 229-238.

Huebner, E. S. (1991b). Correlates of life satisfaction in children. School Psychology Quarterly, 6(2), 103-111.

Huebner, E. S., \& Alderman, G. L. (1993). Convergent and discriminant validation of a children's life satisfaction scale: Its relationship to self- and teacher-reported psychological problems and school functioning. Social Indicators Research, 30 (1), 71-82.

Huebner, E. S., \& Dew, T. (1993). Is life satisfaction multidimensional? The factor structure of the perceived life satisfaction scale. Journal of Psychoeducational Assessment, 11 (4), 345-350.

Huebner, E. S., Laughlin, J. E., Ash, C., \& Gilman, R. (1998). Further val idation of the multidimensional students' life satisfaction scale.J ournal of Psychoeducational Assessment, 16 (2), 118-134.

Hutz, C. S. (2000). Adaptação brasileira da escala de autoestima de Rosenberg. Manuscrito não-publicado, Curso de Pós-Graduação em Psicologia do Desenvolvimento, Universidade Federal do Rio Grande do Sul, Porto Alegre.

Hutz, C. S., \& Giacomoni, C. H. (2000). Adaptação brasileira do inventário de depressão infantil (CDI). Manuscrito não-publicado, Curso de Pós-Graduação em Psicologia do Desenvolvimento, Universidade Federal do Rio Grande do Sul, Porto Alegre.

Kaiser, H. F. (1960). The application of electronic computers to factor analysis. Educational and Psychological Measurement, 20 (1), 141-151.

Kovacs, M. (1980/1981). Rating scales to assess depression in school-aged children. Acta Paedopsychiatrica, 46 (5-6), 305-316.

Kovacs, M. (1983). Thechildren's depression inventory: a self-rated depression scale for school age youngsters. Pittsburg, PA: University of Pittsburgh.

Kovacs, M. (1992). Children's Depression Inventory Manual. Los Angels: Western Psychological Services.

Laurent, J., Catanzaro, S. J., Joiner, T. E., Rudolph, K. D., Potter, K. I., Lambert, S., Osborne, L., \& Gathright, T. (1999). A measure of positive and negative affect for children: Scale development and preliminary validation. Psychological Assessment, 11 (3), 326-338. 
Marsh, H. W. (1986). Negative item bias in rating scales for preadolescent children: A cognitive-developmental phenomenon. Developmental Psychology, 22 (1), 37-49.

Pasquali, L. (1997). Psicometria: teoria e aplicações. Brasília: Editora Universidade de Brasília.

Pasquali, L. (1999). Testes referentes a construto: teoria e modelo de construção. In L. Pasquali (Org.),Instrumentos psicológicos: manual prático de elaboração (pp.37-71). Brasília: LabPAM.

Pavot, W., Diener, E., Colvin, C. R., \& Sandvik, E. (1991) Further validation of the satisfaction with life scale: evidence for the cross-method convergence of well-being measures. Journal of Personality Assessment, 57 (1): 149-161.

Reppold, C. T. (2001). Estilo parental percebido e adaptação psicológica de adolescentes adotados. Dissertação de mestrado não-publicada, Universidade Federal do Rio Grande do Sul, Porto Alegre.
Rosenberg, M. (1965). Society and theadolescent self-image. Princeton: Princeton University Press.

Silva, D. F. M. (2001). O desenvolvimento das trajetórias do comportamento delinqüente em adolescentes infratores. Manuscrito não-publicado, Universidade Federal do Rio Grande do Sul, Porto Alegre.

Spielberger, C. D. (1983). Manual do inventário deansiedade traço-estado-C. Rio de J aneiro: CEPA.

Spielberger, C. D., Gorsuch, R. L., \& Lushene, R. E. (1979). Manual do inventário de ansiedade traço-estado. Rio de Janeiro: CEPA.

Terry, T., \& Huebner, E. S. (1995). The relationship between self-concept and life satisfaction in children. Social Indicators Research, 35 (1), 39-52.

Recebido em: 18/8/2006

Versão final reapresentada em:23/2/2007

Aprovado em:22/3/2007 
